\title{
Recent advances in magnetic resonance neuroimaging of lumbar nerve to clinical applications: A review of clinical studies utilizing Dffusion Tensor Imaging and Diffusion-weighted magnetic resonance neurography
}

\author{
Yawara Eguchi $^{1)}$, Hirohito Kanamoto's), Yasuhiro Oikawa ${ }^{3)}$, Munetaka Suzuki ${ }^{1)}$, Hajime Yamanaka ${ }^{1)}$, Hiroshi Tamai ${ }^{1)}$ \\ Tatsuya Kobayashi ${ }^{1}$, Sumihisa Orita ${ }^{2)}$, Kazuyo Yamauchi ${ }^{2)}$, Miyako Suzuki ${ }^{2)}$, Kazuhide Inage ${ }^{2)}$, Yasuchika Aoki ${ }^{4)}$, \\ Atsuya Watanabe ${ }^{4)}$, Takeo Furuya ${ }^{2)}$, Masao Koda ${ }^{2)}$, Kazuhisa Takahashi ${ }^{2}$ and Seiji Ohtori ${ }^{2)}$ \\ 1) Department of Orthopedic Surgery, National Hospital Organization Shimoshizu National Hospital, Japan \\ 2) Department of Orthopedic Surgery, Graduate School of Medicine, Chiba University, Japan \\ 3) Division of Orthopaedic Surgery, Chiba Children's Hospital, Japan \\ 4) Department of Orthopaedic Surgery, Eastern Chiba Medical Center, Japan
}

\begin{abstract}
:
Much progress has been made in neuroimaging with Magnetic Resonance neurography and Diffusion Tensor Imaging (DTI) owing to higher magnetic fields and improvements in pulse sequence technology. Reports on lumbar nerve DTI have also increased considerably.

Many studies have shown that the use of DTI in lumbar nerve lesions, such as lumbar foraminal stenosis and lumbar disc herniation, makes it possible to capture images of interruptions of tractography at stenotic sties, enabling the diagnosis of stenosis. DTI can also reveal significant decreases in fractional anisotropy (FA) with significant increases in apparent diffusion coefficient (ADC) values in compression lesions.

FA values have higher accuracy than ADC values. Furthermore, strong correlations exist between FA values and indications of neurological severity, including the Japanese Orthopedic Association (JOA) score, the Oswestry Disability Index (ODI), and the Roland-Morris Disability Questionnaire (RDQ) in patients with lumbar disc herniation-induced radiculopathy.

Most lumbar DTI has become 3T; 3T MRI has made it possible to take high-resolution DTI measurements in a short period of time. However, increased motion artifacts in the magnetic susceptibility effect lead to signal irregularities and image distortion. In the future, high-resolution DTI with reduced field-of-view may become useful in clinical applications, since visualization of nerve lesions and quantification of DTI parameters could allow more accurate diagnoses of lumbar nerve dysfunctions. Future translational studies will be necessary to successfully bring MR neuroimaging of lumbar nerve into clinical use.
\end{abstract}

Keywords:

magnetic resonance imaging, diffusion tensor imaging, diffusion-weighted MR neurography, lumbar nerve, lumbar foraminal stenosis, lumbar disc herniation

Spine Surg Relat Res 2017; 1(2): 61-71 dx.doi.org/10.22603/ssrr.1.2016-0015

\section{Introduction}

With the rapid aging of our society, the number of patients with spinal disorders continues to rise. In the United States alone, 25 million patients complain of lumbar pain, leading to medical costs of over 100 billion dollars annually. Intervertebral disc lesions and lumbar nerve root disorders often cause lower back pain. Pain signals are transmitted from the local site to the peripheral nerves, via the spinal cord to the brain, where they are recognized as pain. In re-

Corresponding author: Yawara Eguchi, yawara_eguchi@yahoo.co.jp

Received: October 26, 2016, Accepted: December 30, 2016

Copyright (C) 2017 The Japanese Society for Spine Surgery and Related Research 


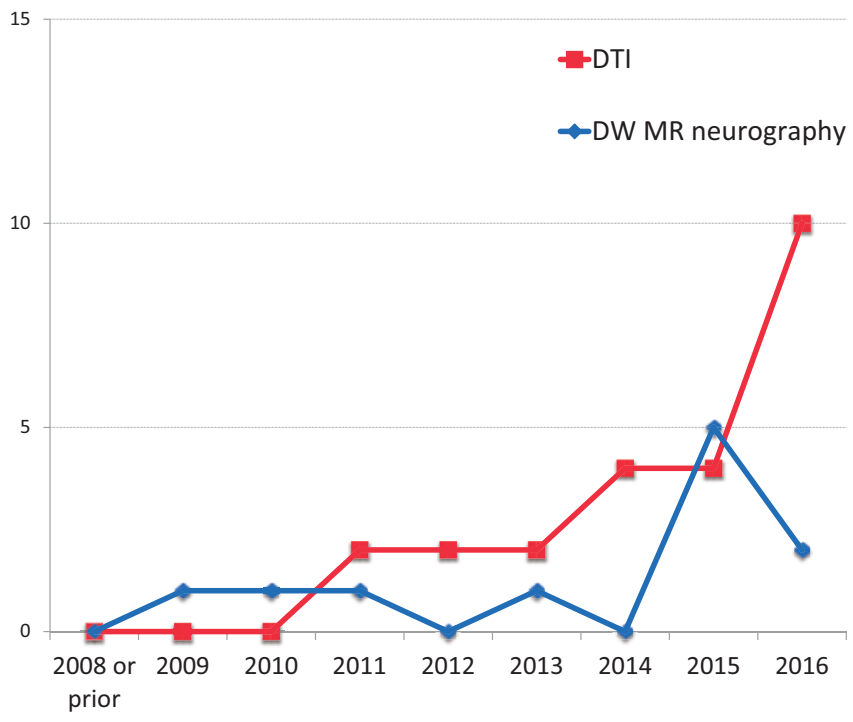

Figure 1. The number of publications per year reporting DTI and DW neurography of the lumbar nerves.

cent years, functional MRI (fMRI) ${ }^{1,2)}$ and MR spectroscopy ${ }^{3)}$ have commonly been employed as neurofunctional imaging methods that involve the brain.

Lumbar nerve entrapment causes low back pain and radiculopathy, but discrepancies are often found between clinical symptoms and the degree of nerve root compression on conventional MR images. For instance, disc abnormalities are frequently observed on images from asymptomatic patients $^{4,5}$, so it has not been possible to understand physiologically the cause of the pain and to provide a quantitative evaluation of nerve damage.

Higher magnetic fields and improvements in pulse sequence technology in recent years have allowed for higherresolution neuroimaging. Conventional 2-dimensional (2D) MR imaging cannot visualize the nerve roots in 1 image, because they cannot be depicted in any optional cross section.

3-dimensional MR neurography is useful for evaluating entire nerves, because it achieves better contrast between nerves and surrounding tissues. Recently, the use of MR neurography has increased for the evaluation of patients with suspected lumbosacral plexus involvement and for aiding in the confirmation of the diagnosis or providing anatomic information should surgical intervention be necessary ${ }^{6}$.

However, this method could only demonstrate morphological abnormalities, such as nerve disruption and narrowing, but it could not quantitatively evaluate nerve damage.

Diffusion weighted imaging (DWI) and diffusion tensor imaging (DTI) are newer MR imaging techniques that measure the in vivo directional coherence of water diffusion. These quantitative techniques can identify pathology that cannot be detected by conventional MR imaging and thus, are important not only for research but also for clinical applications.

This article addresses developments in MR neuroimaging, such as diffusion-weighted MR neurography and DTI of the lumbar nerve in clinical applications.

\section{Trends in clinical MR neuroimaging of lumbar nerves}

We performed a structured search of the PubMed literature database to include relevant articles from 2007 to 2016. The following combinations of search terms were used: "lumbar nerve or lumbosacral plexus and diffusion-weighted MR neurography or DTI." We excluded reviews, spinal cord studies and animal or in vivo studies. Our review identified a total of 35 articles: 24 DTI studies ${ }^{7-30)}$ and 11 diffusionweighted MR neurography studies ${ }^{31-41}$. The number of lumbar nerve DTI studies has sharply increased in recent years (Fig. 1).

A systematic review of spinal cord MRI techniques, including DTI, magnetization transfer (MT), MRS, and fMRI, found that DTI is also being utilized more frequently and is the most mature among these tools ${ }^{42}$.

\section{Diffusion-weighted MR neurography}

DWI based on MRI yields useful information regarding tissue microstructure because it monitors the random movement of water molecules, which is restricted by applying a motion probing gradient (MPG) in some directions ${ }^{43-46}$. DWI data can be used to determine quantitative values, such as the apparent diffusion coefficient (ADC). DWI is frequently used to diagnose central nervous system diseases, such as acute stroke ${ }^{47}$. It is also useful for the evaluation and diagnosis of lesions, such as those in multiple sclerosis ${ }^{48,49)}$ and peripheral nerve compression disorders, such as carpal tunnel syndrome ${ }^{50-54)}$. Increased mean ADC values were observed in injured nerves with demyelination ${ }^{48,49,52,53)}$. Spinal cord imaging has technical limitations, such as the relatively small size of the cord, susceptibility artifacts from tissuebone interfaces, and motion artifacts from respiratory activity $^{54)}$. Yamashita and Takahara et al. ${ }^{55)}$ (Fig. 2A) demonstrated the feasibility of whole-body MR neurography with DWI that can visualize tissues with impeded diffusion, such as tumors, the brain, the spinal cord, and the peripheral nerves ${ }^{56)}$. We found that diffusion-weighted MR neurography revealed that nerve swelling and transverse and mean ADC values were significantly greater in entrapped nerve roots in patients with lumbar disc herniation ${ }^{39)}$ and lumbar foraminal stenosis (Fig. 2B, C) ${ }^{40)}$, suggesting demyelination and edema by slow compression caused by an increased degree of diffusion compared with that of normal tissue.

However, this method requires restructuring of $3 \mathrm{D}$ nerve images with maximum intensity projection (MIP) processing which is a volume rendering method for $3 \mathrm{D}$ imaging that projects the voxels with maximum intensity of the axial image, which leads to limitations in image resolution. 

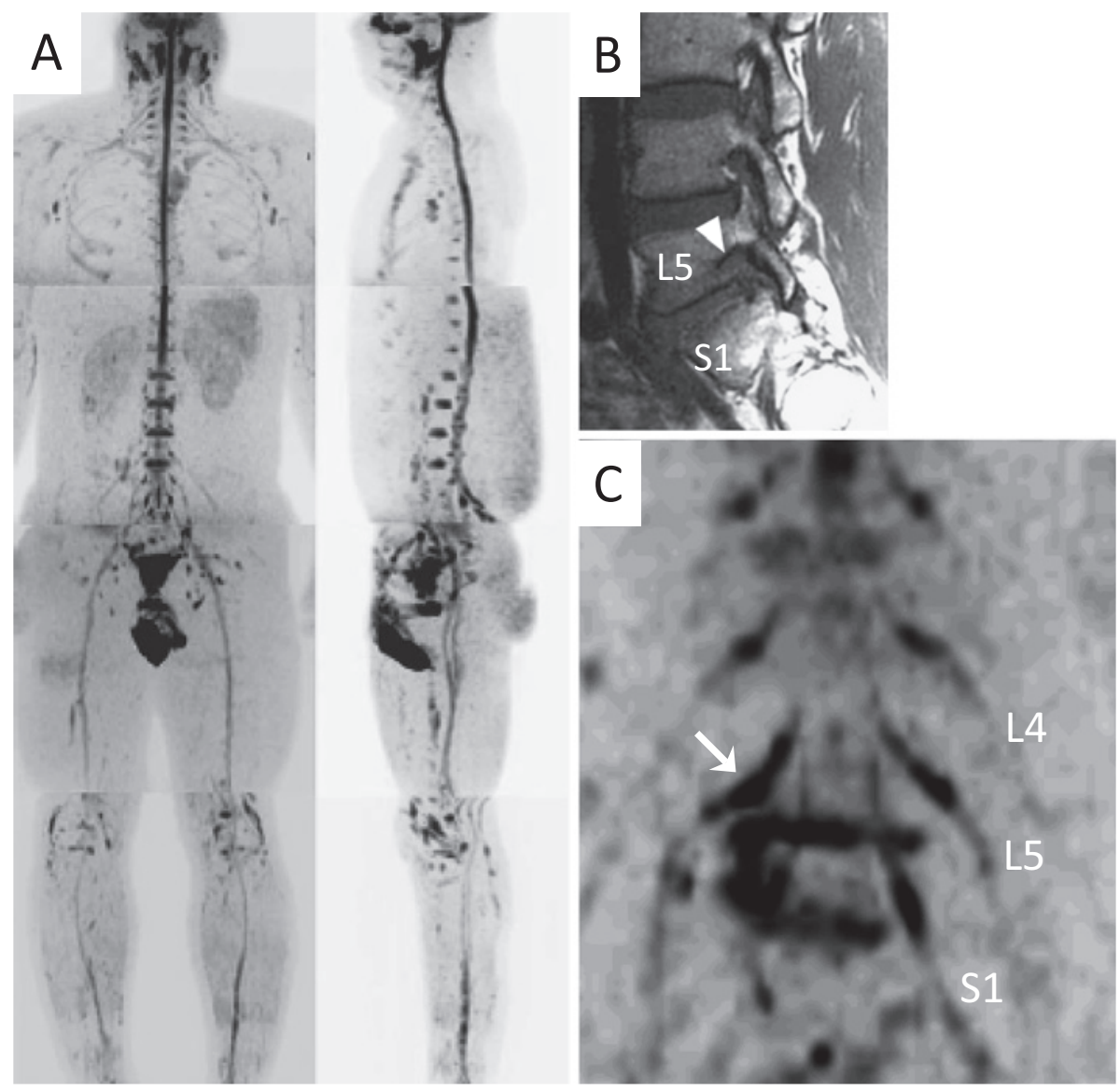

Figure 2. Whole-body MR neurography in coronal (left) and sagittal (right) views in a healthy volunteer (A). Taken from Yamashita et al. ${ }^{60}$ ) with permission from the Massachusetts Medical Society. Parasagittal T1-weighted image (B) and coronal neurography using $\mathrm{b}=1000$ image (C) of a lumbar nerve root in a 66 year old man with L5-S1 foraminal stenosis. The arrowhead shows L5 foraminal stenosis with loss of the perineural fat signal (B). The arrow shows the entrapped nerve shifted upward and ran transversely in the foramen (C).

\section{Diffusion Tensor Imaging (DTI)}

DWI has a strong effect on not only the ease but also the directionality of water molecule dispersion. Axonal cell membranes and myelinated sheaths prevent dispersion in the direction directly aligned to the nerve fiber bundle, which leads to a loss of water molecule isotropy. This condition is referred to as anisotropy, and DTI and tractography are examples of selective recording of this data. FA is a parameter of anisotropic strength and is expressed in values from 0 to 1 . The closer the value is to 1 , the stronger the anisotropy, while a value of 0 is complete isotropy. DTI has recently been reported to be useful in demyelinating diseases, such as multiple sclerosis, or for chronic peripheral nerve compression lesions, such as carpal tunnel syndrome ${ }^{51)}$. Water molecule dispersion due to myelin sheaths in myelinated nerves in tissues occurs only in parallel to the nerve fibers, revealing high anisotropy, but when demyelination occurs as a result of nerve injuries such as spinal cord injury, the anisotropy decreases, leading to decreases in FA values.

\subsection{Subjects of DTI lumbar nerve studies}

Table 1 summarizes recent DTI lumbar nerve studies ${ }^{7-30}$. A total of 683 subjects were studied across 24 DTI articles. 177 patients with lumbar spinal canal stenosis (LSCS) in 5 $\operatorname{articles}^{11,12,16,17,20)}, 143$ patients with lumbar disc herniation (LDH) in 7 articles $^{7,13,15,20,21,23,29)}$, 112 patients with lumbar radiculopathy in 3 articles $^{10,19,22)}, 36$ patients with foraminal stenosis (FS) in 6 articles $^{911,16,20,27,28}, 2$ patients with lower back pain in one article $e^{20}, 3$ cases with diastematomyelia and one case with tethered cord in one article ${ }^{30)}$, and one case with spina bifida in one article ${ }^{26)}$ were included. 7 post mortem subjects were investigated in one article $^{8)} .201$ healthy volunteers were also investigated in 15 articles $^{8,11,14,16-19,22-26,28-30)}$.

\subsection{Measurement of DTI parameters}

FA values were examined in all studies. All 17 articles $^{7,9-13,15-17,19,2,22,2,2,27-30)}$ demonstrated findings of low FA in the compressed nerves. ADC is the next most commonly reported parameter in 16 articles $^{7-1,1,13,15,16,18,21-26,29)}$. Except for one 


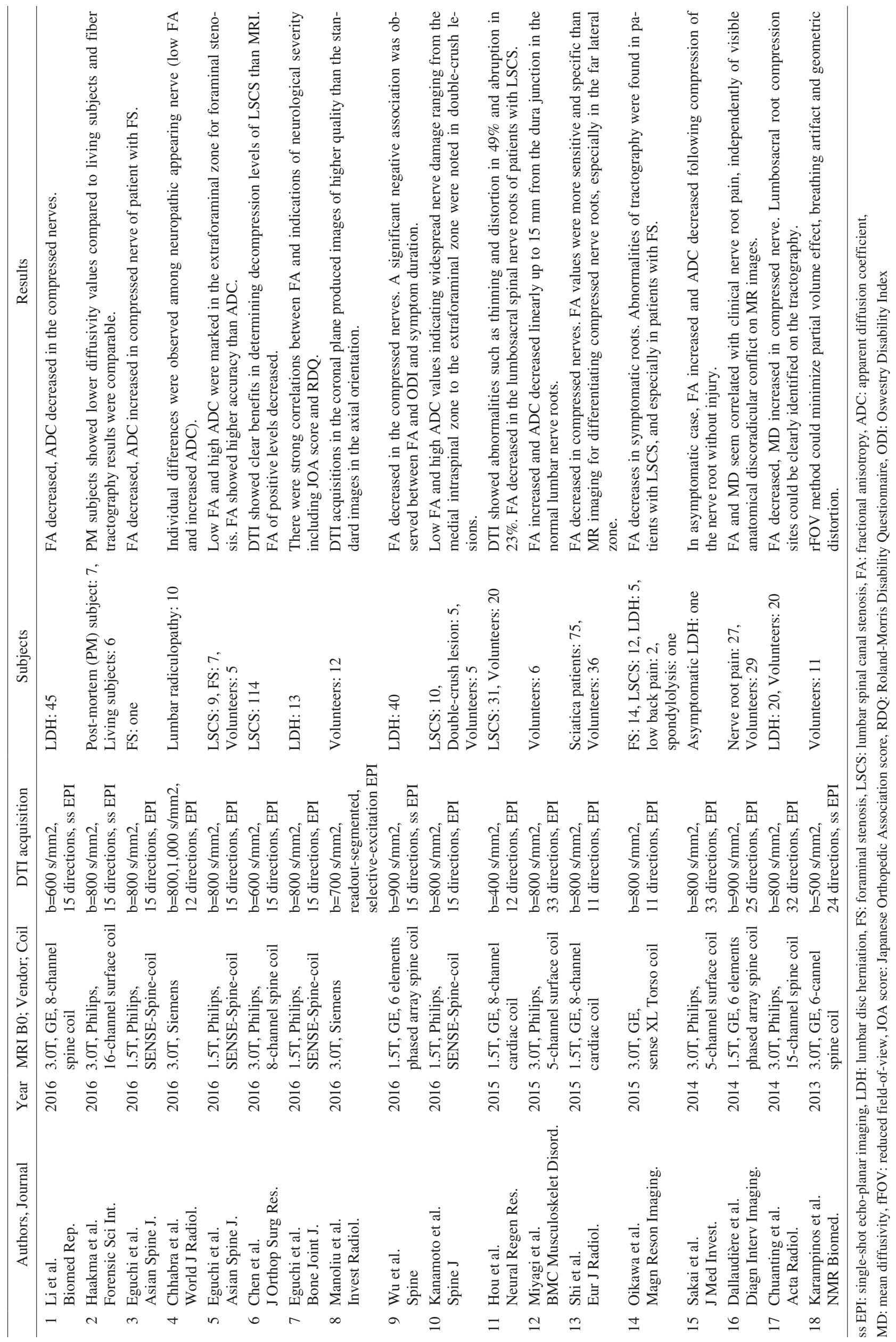




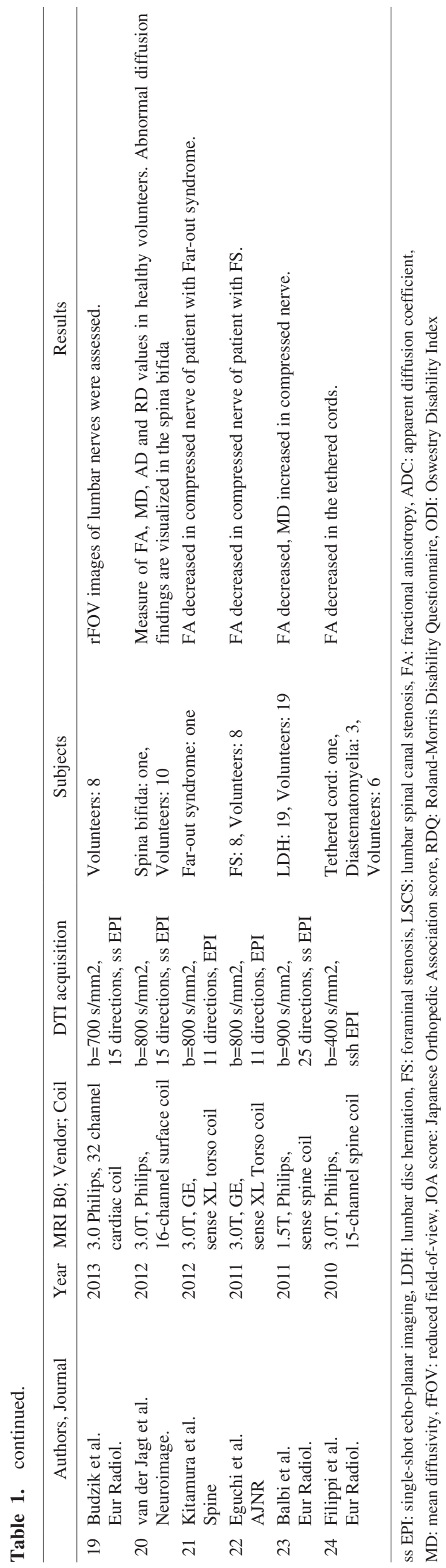

$\operatorname{article}^{7)}$ (which showed low ADC), 9 articles $^{9-11,13,15,16,22,23,29)}$ described high ADC in involved nerves and 5 articles demonstrated findings of ADC in healthy subjects ${ }^{8,18,24-26)}$. In an asymptomatic case, FA increased and ADC decreased following compression of the nerve root without injury ${ }^{21}$.

Takagi et al. ${ }^{57)}$ demonstrated that the FA values of the peripheral nerves were more strongly correlated with the axonal degeneration and regeneration in rat and mouse sciatic nerves. Generally, increased ADC may be due to inflammation or oedema, whereas decreased FA may reflect damaged tissue microstructure, demyelination, axonal loss, or increase in isotropic water volume ${ }^{57}$. A systematic review of spinal cord MRI techniques including DTI found that FA value has the most robust support for clinical utility ${ }^{42)}$.

\subsection{DTI acquisition parameters}

Parameters including magnetic field strength, motionprobing gradient (MPG), and b-values are important in the clinical use of DTI. High magnetic field strengths have the advantages of greater signal-to-noise ratio, improved spatial resolution, and faster imaging times, but also have the disadvantage of the magnetic susceptibility artifact. Magnetic susceptibility artifact indicated MRI artifacts that share distortions due to local inhomogeneous magnetic field. Fifteen reports used 3T, and 9 used $1.5 \mathrm{~T}$, so it appears that $3 \mathrm{~T}$ research accounted for the majority.

The methods of measuring in vivo diffusion by MRI have been based mainly on the addition of several MPGs to the spin echo sequence to produce signal attenuation for the spins moving at random. Additional MPG directions can increase the resolution, but will prolong the scan time. MPG directions ranged from 11 to 33 , where 15 directions was most common, used in 10 reports.

The b-value is a factor that defines gradient strength and duration and determines the degree of diffusion weighted images typically using values from 0 to $1000 \mathrm{~s} / \mathrm{mm}^{2}$. The higher the b-value, the stronger diffusion effects. Increasing the $b$-value increases the diffusion sensitivity, but decreases the signal-to-noise ratio. B-values ranged between 400 and 900 , with $b=800$ the most common, used in 14 reports (Table 1).

\subsection{Diagnosis of lumbar foraminal stenosis with DTI}

In patients with lumbar foraminal stenosis, a nerve root or spinal nerve is entrapped in a narrowed lumbar foramen as the result of a degenerative lumbar spinal disorder. At this site, a dorsal root ganglion functions as a pain receptor; thus, the condition is refractory and may cause severe lower limb pain $^{58)}$. Macnab et al. ${ }^{59)}$ appropriately named lumbar foraminal stenosis the "hidden zone," as it is often overlooked, it accounts for approximately $60 \%$ of failed back surgery syndromes, and it decreases surgical success rates ${ }^{60)}$.

Diagnostic imaging of lumbar spinal canal stenosis includes X-rays, CT, and $\mathrm{MRI}^{61-63)}$, along with functional diagnosis via selective nerve root imaging and infiltration ${ }^{64}$. Conventional MRI has a false positive rate of $30 \%$ to $40 \%$ 

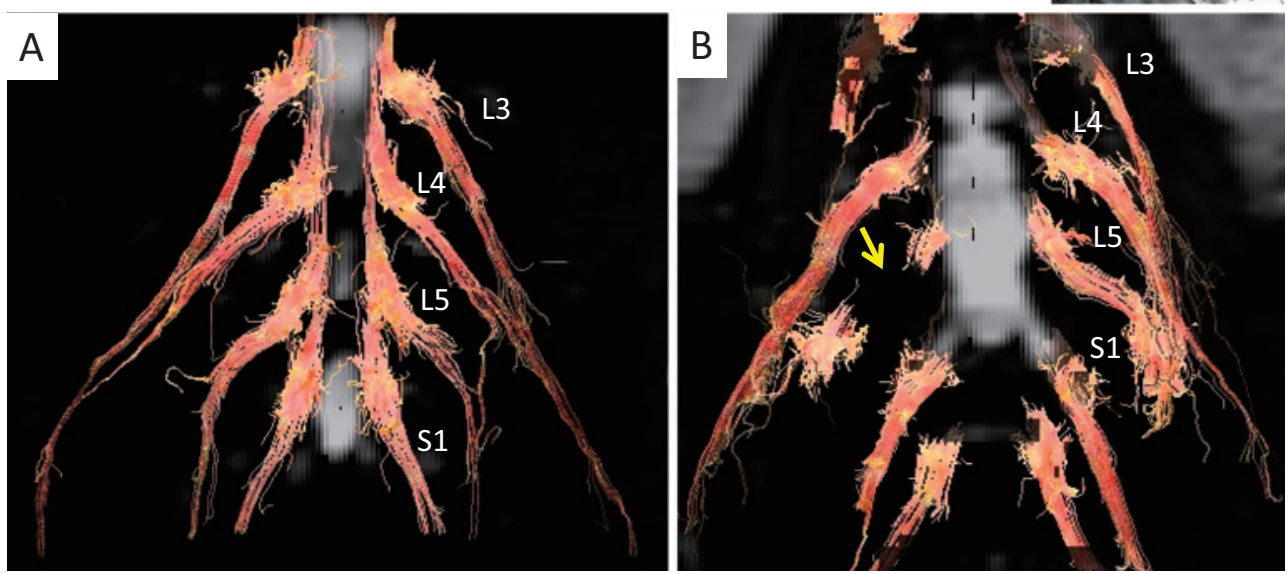

Figure 3. Coronal tractography of a lumbar nerve in a healthy volunteer (A). L3, L4, L5, and S1 indicate the third, fourth, and fifth lumbar root, and the first sacral root, respectively. Tractography (B) and parasagittal T1-weighted image (C) of a lumbar nerve root in a 75 year old man with right L5-S1 foraminal stenosis. Tractography showed disruption in the course through the foramen.

in lumbar foraminal stenosis cases; thus, diagnosis is difficult $^{65)}$. Recently 3D-CT, MR myelography ${ }^{65,66)}$, and 3D-MRI ${ }^{67}$ were reported to be useful for diagnosis.

We assessed the usefulness of DTI for the diagnosis of lumbar foraminal stenosis. Subjects in a supine position were scanned with a 3T MR imaging scanner (Discovery MR750; GE Healthcare, Milwaukee, WI) using a Sense XL Torso coil. We performed DTI with echo-planar imaging at a B value of $800 \mathrm{~s} / \mathrm{mm}^{2}$ and with 11 directional MPGs. Mean FA values and mean ADC values in the lumbar nerve roots were quantified on DTI images, and the lumbar nerve roots were visualized with tractography. In all subjects, the lumbar nerve roots were clearly visualized (Fig. 3A), and tractography also showed abnormalities, such as tract disruption, nerve narrowing, and indentation in their course through the foramen (Fig. 3B). Mean FA values were significantly lower, and mean ADC values were significantly higher, in entrapped versus intact roots ${ }^{28)}$.

We evaluated the accuracy of these parameters for the diagnosis of foraminal stenosis. Changes in DTI parameters (low FA and high ADC values) were marked in nerve roots and in the extraforaminal zone in foraminal stenosis. Additionally, the FA value was more accurate than the ADC value in the extraforaminal zone, and a low FA value suggested foraminal stenosis ${ }^{11}$.

L5 radiculopathy may occur when the nerve is compressed at two levels (medial and lateral), for example, when the L4/5 level is compressed by an intraspinal canal lesion and the L5/S1 level is compressed by a lateral lesion. This is called a double-crush lesion. Traditional imaging studies do not allow a determination of whether the compressing lesion is inside or outside of the spinal canal, or if a doublecrush lesion is responsible, which leads to poor surgical success rates. The distal latency of L5 has been measured electrophysiologically; however, this is an invasive approach, and noninvasive diagnostic methods are virtually nonexistent ${ }^{68,69)}$. Diagnosis of L5 nerve damage double-crush lesions by DTI was studied. Low FA values and high ADC values indicative of widespread nerve damage from the medial intraspinal zone to the extraforaminal zone were found for double-crush lesions. If double-crush injury is suspected prior to surgery, DTI assessment may help prevent failed back surgery syndrome ${ }^{16)}$ (Fig. 4).

\subsection{DTI evaluation of radiculopathy in patients with lum- bar disc herniation}

Mixter and $\mathrm{Barr}^{70)}$ first described radicular pain of the sciatica as spinal root compression by a herniated intervertebral disc; however, the underlying pathophysiology is not well understood. In clinical practice, asymptomatic intervertebral disc degeneration and herniation are often found, and these discrepancies can confuse spine surgeons ${ }^{4,5}$. We previously reported a correlation between neurological severity and DTI parameters, such as the FA and ADC values, in patients with radiculopathy caused by lumbar disc herniation ${ }^{13)}$. Strong correlations were found between the FA value and indications of neurological severity, including the Japanese Orthopedic Association (JOA) score, the Oswestry Disability In- 

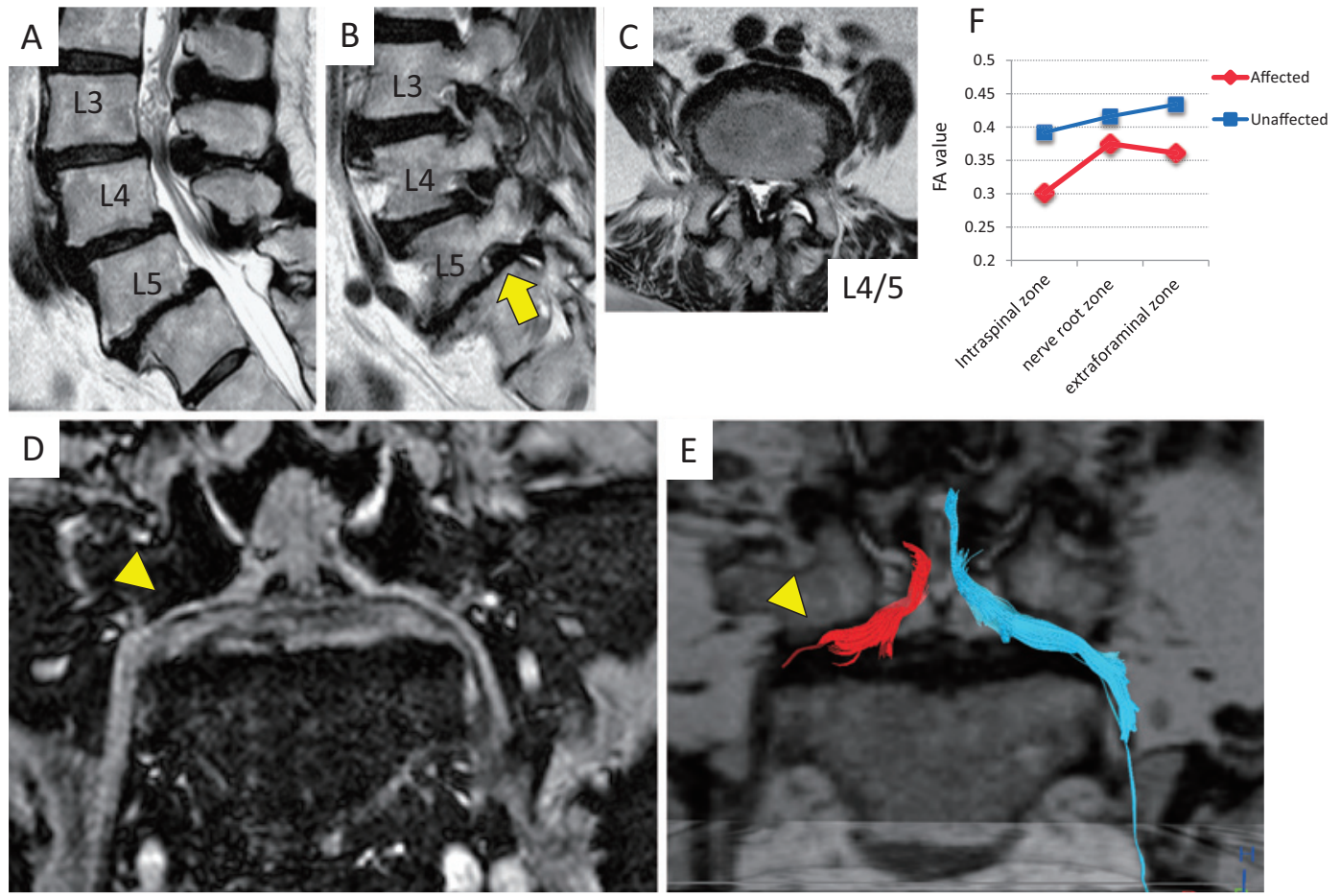

Figure 4. Presurgical lumbar MRI images and tractography of an 87 year old woman with double-crush lesions. She complained of persistent symptoms due to L5 foraminal stenosis despite L4/5 decompression surgery. A) Sagittal T2-weighted image B) Right parasagittal T1-weighted image. Right L5 foraminal stenosis (arrow) can be noted. C) L3/4 level axial image D) Coronal 3D-MR image. Right L5 nerve swelling and running transversely in the course through the foramen (arrowhead). E) Fusion image of lumbar DTI images and 3D-T2 weighted images. The right L5 nerve is disrupted at the foraminal area (arrowhead). F) FA values (intraspinal zone, nerve root zone, and extraforaminal zone) were $0.301,0.375$, and 0.361 , respectively, on the affected side and $0.392,0.416$, and 0.434 , respectively, on the unaffected side. FA values were decreased over a widespread area from the intraspinal to the extraforaminal zone.
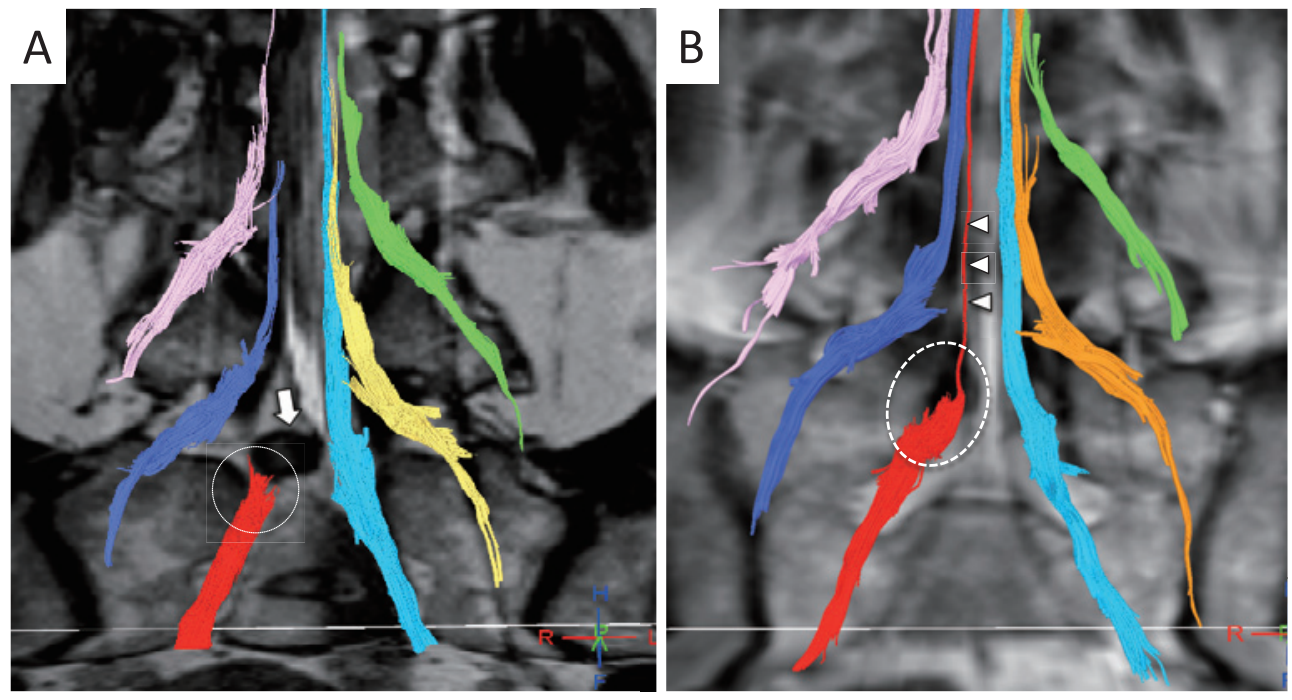

Figure 5. Tractography of lumbar nerves in a patient with lumbar disc herniation between the L5 and S1 discs before and after microendoscopic discectomy (MED). Tractography of the S1 nerve on the right side was disrupted by disc herniation (arrow) before MED. At six months after MED, leg numbness was decreased from 60 to 10 , the FA value was increased from 0.299 to 0.327 , and the ADC value was decreased from $1.173 \mathrm{~mm}^{2} / \mathrm{s}$ to $1.096 \mathrm{~mm}^{2} / \mathrm{s}$. Tractography of the $\mathrm{S} 1$ nerve was elongated to the proximal side (arrowheads). 

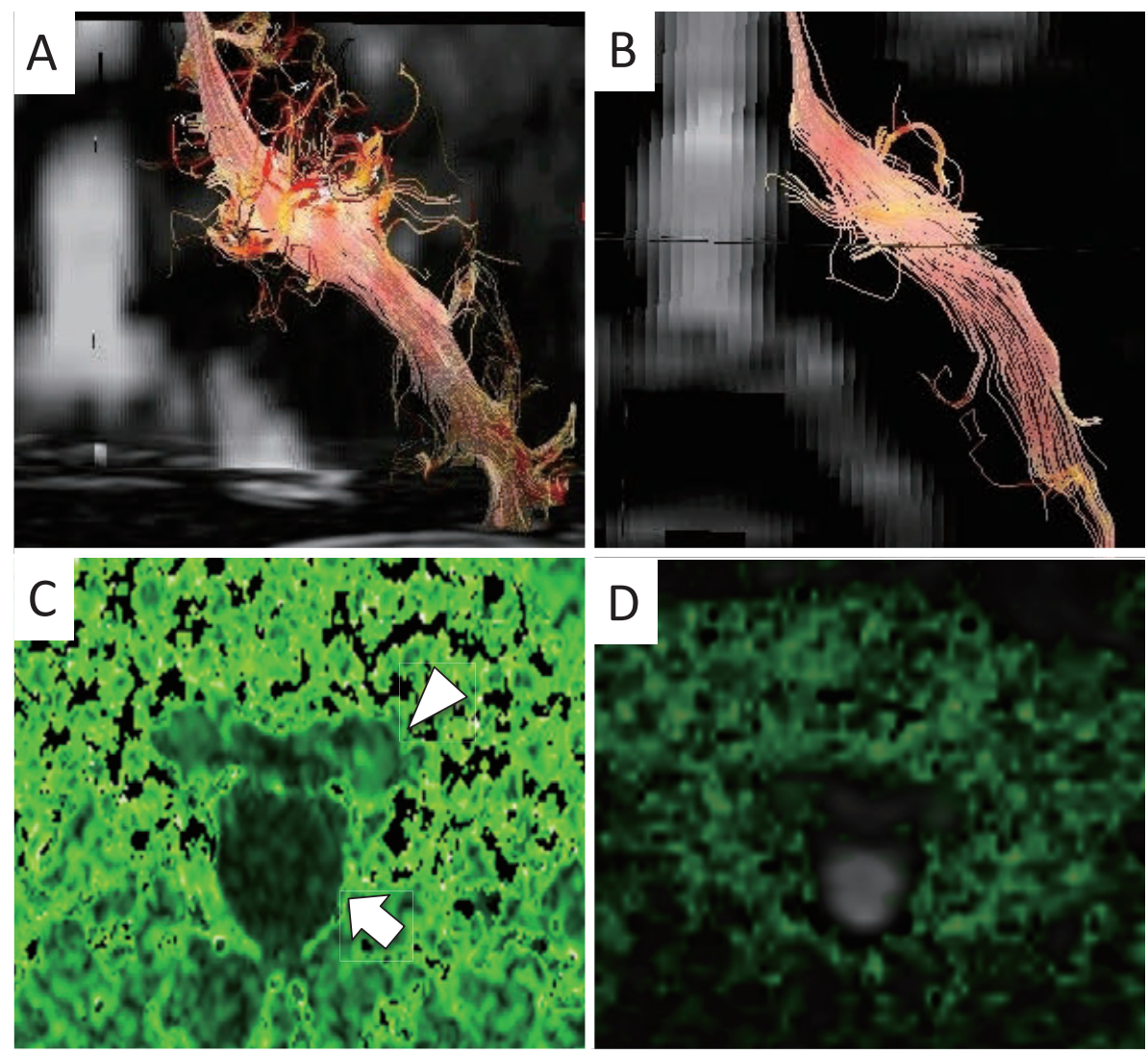

Figure 6. Tractography of the L5 and $\mathrm{S} 1$ nerves in a healthy volunteer with reduced FOV (rFOV) (A) and conventional FOV (cFOV) (B). Axial FA map at the L4/5 level in a healthy volunteer with $\mathrm{rFOV}(\mathrm{C})$ and $\mathrm{cFOV}(\mathrm{D})$. Fiber counts are higher with $\mathrm{rFOV}$ versus cFOV, allowing for clearer imaging of the lumbar nerve. FA map resolution is higher with rFOV allowing for clearer imaging of the nerve root (arrow head) and the spinal canal (arrow).

dex $(\mathrm{ODI})^{15)}$, and the Roland-Morris Disability Questionnaire $(\mathrm{RDQ})^{13)}$. DTI parameters are useful for diagnosis, quantitative assessment, and follow-up of lumbar nerve entrapment (Fig. 5).

Advantages and limitations of DTI

It has not been possible to understand the physiological cause of pain by conventional MR images because of the discrepancy between clinical symptoms and MRI findings, which may be an issue for diagnostic and therapeutic management. Conventional MR imaging also cannot selectively visualize peripheral nerves and cannot quantitatively assess the severity of the nerve lesion. DTI is a non-invasive way to effectively trace the nerve fiber bundle, and quantitatively evaluate the nerve injury.

DTI has several limitations. Firstly, tractography is mathematical modeling of the diffusion tensor data using probability theories to model the most likely course of diffusion, and the number of tracts visualized by DTI did not present the actual volume of nerve fiber trajectories. Second, 3T MRI has made it possible to take high-resolution DTI measurements in a short period of time. However, since it is a high magnetic field, the magnetic susceptibility effect and increases in motion artifacts lead to signal irregularities and image distortion so that nerve fiber follow-up is limited in areas with artifacts. Third, the evidence is insufficient to support DTI as a diagnostic tool or predictor of clinical outcomes. Fourth, automatic analysis methods such as tractspecific automatic ROI placement are needed.

Further translational research is required to link pathophysiologic mechanisms in animal models to clinical outcomes in patients.

\subsection{Future directions; High-resolution DTI}

In the future, further improvements in image resolution are essential for clinical applications. A new reduced fieldof-view FOV (rFOV) single-shot diffusion-weighted echoplanar imaging method that uses a $2 \mathrm{D}$ spatially selective echo-planar RF excitation pulse and a $180^{\circ}$ refocusing pulse to reduce the FOV in the phase-encode (PE) direction, and simultaneously suppresses the signal from fat has been proposed. The rFOV method decreases the readout duration and allows acquisition of high-resolution diffusion-weighted images for practical application to spinal imaging ${ }^{71}$.

rFOV DTI of the spinal cord allows for acquisition of high-resolution images for assessment of specific spinal cord tracts and discrimination of white matter from gray matter. Maki et al. ${ }^{72)}$ showed correlations between the FA of specific white matter in spinal cord tracts and myelopathy severity in 

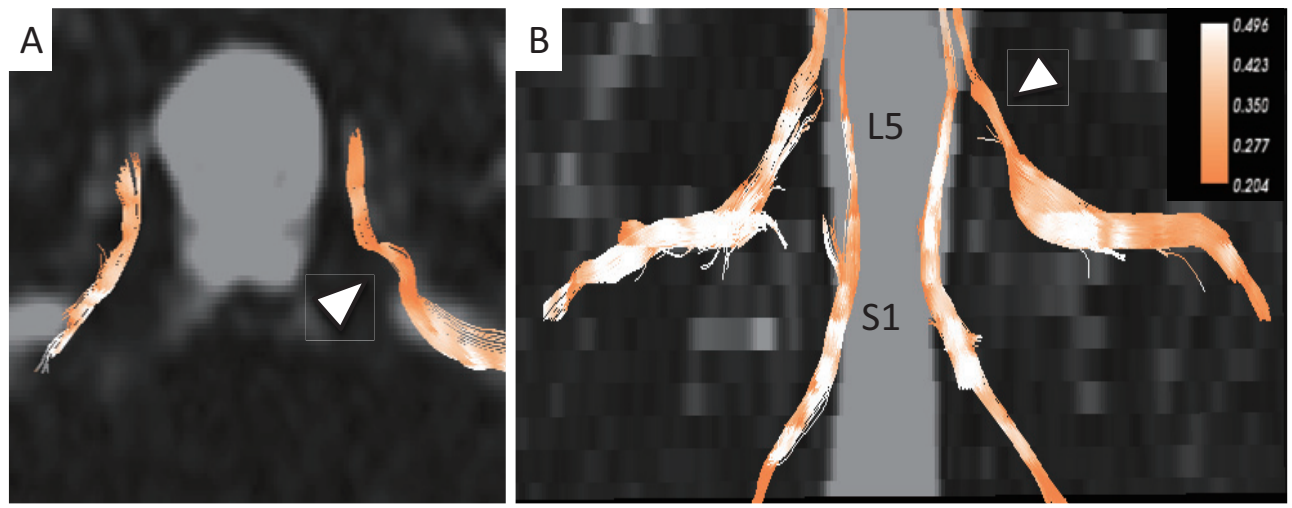

Figure 7. rFOV tractography of a patient with left L5 intervertebral foraminal encroachment using TrackVis. Axial image (A) and coronal image of L5 foramina showed signs of nerve pinching (arrowhead) (B). No injury (FA value normal: white) to injury (FA value decreased: orange) and FA value changes are expressed in colors allowing for visualization around the compressed region in orange (decreased FA) (C).

patients with cervical compression myelopathy with rFOV DTI. Several authors ${ }^{24,25)}$ reported that $\mathrm{rFOV}$ imaging was necessary for lumbar nerve root DTI imaging and tractography in 3T, as it drastically reduced the susceptibility and chemical shift artifacts that hindered accurate tractography on conventional FOV imaging.

We attempted high-resolution imaging of the lumbar nerves with rFOV 3T MRI. Compared to traditional methods, rFOV allows for clear imaging of the lumbar nerves and enables accurate measurements of the FA and ADC values (Fig. 6A-D). By expressing consecutive FA changes in color, we found that it was possible to visualize the degree of nerve damage (Fig. 7). This method allows for direct visualization of consecutive changes in FA values, and it may break through the limitations of ROI establishment such as the partial volume effect.

In the future, high-resolution DTI with rFOV may be used to visualize nerve lesions and allow for more accurate diagnosis of DTI parameter quantification with opportunities for clinical applications.

\section{Conclusions}

Recent advances have allowed the use of higher magnetic fields and pulse sequencing improvements in MRI devices that provide better neuroimaging, such as DTI in the lumbar nerves. DTI of spinal nerves is more likely to be affected by the magnetic rate than the brain; therefore, from a technical viewpoint, it can hardly be considered a widely employed test in the field, but reports continue to suggest that it may reveal information that is not available on a conventional MRI.

In the future, introduction of high-resolution DTI will allow more detailed visualization of lumbar nerve lesions that may be quantified, and we look forward to further expansion in the functional diagnosis of lumbar nerve disorders. Future translational studies will be essential to successfully bring these quantitative techniques into clinical use.
Conflicts of Interest: The authors declare that there are no conflicts of interest.

\section{References}

1. Ogawa S, Lee TM, Kay AR, Tank DW. Brain magnetic resonance imaging with contrast dependent on blood oxygenation. Proc Natl Acad Sci USA 1990; 87(24): 9868-72.

2. Kobayashi Y, Kurata J, Sekiguchi M, et al. Augmented cerebral activation by lumbar mechanical stimulus in chronic low back pain patients: an FMRI study. Spine 2009; 34(22): 2431-6.

3. Shigemura T, Kishida S, Eguchi Y, et al. Proton magnetic resonance spectroscopy of the thalamus in patients with osteoarthritis of the hip. Bone Joint Res 2012; 1: 8-12.

4. Boden SD, Davis DO, Dina TS, Patronas NJ, Wiesel SW. Abnormal magnetic-resonance scans of the lumbar spine in asymptomatic subjects. A prospective investigation. J Bone Joint Surg Am 1990; 72(3): 403-8.

5. Jensen MC, Brant-Zawadzki MN, Obuchowski N, Modic MT, Malkasian D, Ross JS. Magnetic resonance imaging of the lumbar spine in people without back pain. N Engl J Med 1994; 331(2): 69-73.

6. Soldatos T, Andreisek G, Thawait GK, et al. High-resolution 3-T MR neurography of the lumbosacral plexus. Radiographics 2013; 33(4): 967-87.

7. Li J, Wang Y, Wang Y, Lv Y, Ma L. Study on lumbosacral nerve root compression using DTI. Biomed Rep 2016; 5(3): 353-6.

8. Haakma W, Pedersen M, Froeling M, Uhrenholt L, Leemans A, Boel LW. Diffusion tensor imaging of peripheral nerves in nonfixed post-mortem subjects. Forensic Sci Int 2016; 263: 139-46.

9. Eguchi Y, Ohtori S, Suzuki M, et al. Diagnosis of lumbar foraminal stenosis using diffusion tensor imaging. Asian Spine J 2016;10 (1): 164-9.

10. Chhabra A, Farahani SJ, Thawait GK, Wadhwa V, Belzberg AJ, Carrino JA. Incremental value of magnetic resonance neurography of Lumbosacral plexus over non-contributory lumbar spine magnetic resonance imaging in radiculopathy: A prospective study. World J Radiol 2016; 8(1): 109-16.

11. Eguchi Y, Ohtori S, Suzuki M, et al. Discrimination between lumbar intraspinal stenosis and foraminal stenosis using diffusion tensor imaging parameters: preliminary results. Asian Spine J 2016; 10(2): 327-34.

12. Chen HB, Wan Q, Xu QF, Chen $Y$, Bai B. Reducing surgical lev- 
els by paraspinal mapping and diffusion tensor imaging techniques in lumbar spinal stenosis. J Orthop Surg Res 2016; 11(1): 47.

13. Eguchi Y, Oikawa Y, Suzuki M, et al. Diffusion tensor imaging of radiculopathy in patients with lumbar disc herniation: preliminary results. Bone Joint J 2016; 98-B(3): 387-94.

14. Manoliu A, Ho M, Nanz D, et al. Diffusion tensor imaging of lumbar nerve roots: comparison between fast readout-segmented and selective-excitation acquisitions. Invest Radiol 2016; 51(8): 499-504.

15. Wu W, Liang J, Ru N, et al. Microstructural changes in compressed nerve roots are consistent with clinical symptoms and symptom duration in patients with lumbar disc herniation. Spine 2016; 41(11): E661-6.

16. Kanamoto H, Eguchi Y, Suzuki M, et al. The diagnosis of doublecrush lesion in the L5 lumbar nerve using diffusion tensor imaging. Spine J 2016; 16(3): 315-21.

17. Hou ZJ, Huang Y, Fan ZW, Li XC, Cao BY. Changes in lumbosacral spinal nerve roots on diffusion tensor imaging in spinal stenosis. Neural Regen Res 2015; 10(11): 1860-4.

18. Miyagi R, Sakai T, Yamabe E, Yoshioka H. Consecutive assessment of FA and ADC values of normal lumbar nerve roots from the junction of the dura mater. BMC Musculoskelet Disord 2015; 16: 156.

19. Shi Y, Zong M, Xu X, et al. Diffusion tensor imaging with quantitative evaluation and fiber tractography of lumbar nerve roots in sciatica. Eur J Radiol 2015; 84(4): 690-5.

20. Oikawa Y, Eguchi Y, Inoue G, et al. Diffusion tensor imaging of lumbar spinal nerve in subjects with degenerative lumbar disorders. Magn Reson Imaging 2015; 33(8): 956-61.

21. Sakai T, Miyagi R, Yamabe E, Fujinaga Y, N Bhatia N, Yoshioka $\mathrm{H}$. Diffusion-weighted imaging and diffusion tensor imaging of asymptomatic lumbar disc herniation. J Med Invest 2014; 61(1-2): 197-203.

22. Dallaudière B, Lincot J, Hess A, et al. Clinical relevance of diffusion tensor imaging parameters in lumbar disco-radicular conflict. Diagn Interv Imaging 2014; 95(1): 63-8.

23. Chuanting L, Qingzheng W, Wenfeng X, Yiyi H, Bin Z. 3.0T MRI tractography of lumbar nerve roots in disc herniation. Acta Radiol 2014; 55(8): 969-75.

24. Karampinos DC, Melkus G, Shepherd TM, et al. Diffusion tensor imaging and $\mathrm{T} 2$ relaxometry of bilateral lumbar nerve roots: feasibility of in-plane imaging. NMR Biomed 2013; 26(6): 630-7.

25. Budzik JF, Verclytte S, Lefebvre G, Monnet A, Forzy G, Cotten A. Assessment of reduced field of view in diffusion tensor imaging of the lumbar nerve roots at 3 T. Eur Radiol 2013; 23(5): 1361-6.

26. van der Jagt PK, Dik P, Froeling M, et al. Architectural configuration and microstructural properties of the sacral plexus: a diffusion tensor MRI and fiber tractography study. Neuroimage 2012; 62(3): 1792-9.

27. Kitamura M, Eguchi Y, Inoue G, et al. A case of symptomatic extra-foraminal lumbosacral stenosis ("far-out syndrome") diagnosed by diffusion tensor imaging. Spine 2012; 37(14): E854-7.

28. Eguchi Y, Ohtori S, Orita S, et al. Quantitative evaluation and visualization of lumbar foraminal nerve root entrapment by using diffusion tensor imaging: preliminary results. AJNR Am J Neuroradiol 2011; 32(10): 1824-9.

29. Balbi V, Budzik JF, Duhamel A, Bera-Louville A, Le Thuc V, Cotten A. Tractography of lumbar nerve roots: initial results. Eur Radiol 2011; 21(6): 1153-9.

30. Filippi CG, Andrews T, Gonyea JV, Linnell G, Cauley KA. Magnetic resonance diffusion tensor imaging and tractography of the lower spinal cord: application to diastematomyelia and tethered cord. Eur Radiol 2010; 20(9): 2194-9.

31. Ishikawa $\mathrm{T}$, Asakura $\mathrm{K}$, Mizutani $\mathrm{Y}$, et al. Magnetic resonance neurography for the evaluation of CIDP. Muscle Nerve. 2016 in press.

32. Chhabra A, Carrino JA, Farahani SJ, et al. Whole-body MR neurography: Prospective feasibility study in polyneuropathy and Charcot-Marie-Tooth disease. J Magn Reson Imaging 2016 in press.

33. Quinn JC, Fruauff K, Lebl DR, et al. Magnetic resonance neurography of the lumbar plexus at the L4-L5 disc: Development of a preoperative surgical planning tool for lateral lumbar transpsoas interbody fusion (LLIF). Spine 2015; 40(12): 942-7.

34. Menezes CM, de Andrade LM, Herrero CF, et al. Diffusionweighted magnetic resonance (DW-MR) neurography of the lumbar plexus in the preoperative planning of lateral access lumbar surgery. Eur Spine J 2015; 24(4): 817-26.

35. Kasper JM, Wadhwa V, Scott KM, Rozen S, Xi Y, Chhabra A. SHINKEI--a novel 3D isotropic MR neurography technique: technical advantages over 3DIRTSE-based imaging. Eur Radiol 2015; 25(6): 1672-7.

36. Mürtz P, Kaschner M, Lakghomi A, et al. Diffusion-weighted MR neurography of the brachial and lumbosacral plexus: $3.0 \mathrm{~T}$ versus 1.5 T imaging. Eur J Radiol 2015; 84(4): 696-702.

37. Yuh EL, Jain Palrecha S, Lagemann GM, et al. Diffusivity measurements differentiate benign from malignant lesions in patients with peripheral neuropathy or plexopathy. AJNR Am J Neuroradiol 2015; 36(1): 202-9.

38. Yoneyama M, Takahara T, Kwee TC, Nakamura M, Tabuchi $T$. Rapid high resolution MR neurography with a diffusion-weighted pre-pulse. Magn Reson Med Sci 2013; 12(2): 111-9.

39. Eguchi Y, Ohtori S, Yamashita M, et al. Diffusion-weighted magnetic resonance imaging of symptomatic nerve root of patients with lumbar disk herniation. Neuroradiology 2011; 53(9): 633-41.

40. Eguchi Y, Ohtori S, Yamashita M, et al. Clinical applications of diffusion magnetic resonance imaging of the lumbar foraminal nerve root entrapment. Eur Spine J 2010; 19(11): 1874-82.

41. Zhang Z, Song L, Meng Q, et al. Morphological analysis in patients with sciatica: a magnetic resonance imaging study using three-dimensional high-resolution diffusion-weighted magnetic resonance neurography techniques. Spine 2009; 34(7): E245-50.

42. Martin AR, Aleksanderek I, Cohen-Adad J, et al. Translating stateof-the-art spinal cord MRI techniques to clinical use: A systematic review of clinical studies utilizing DTI, MT, MWF, MRS, and fMRI. Neuroimage Clin 2015; 10: 192-238.

43. Basser PJ, Jones DK. Diffusion tensor MRI: theory, experimental design and data analysis-a technical review. NMR Biomed 2002; 15: 456-67.

44. Beaulieu C, Allen PS. Determinants of anisotropic water diffusion in nerves. Magn Reson Med 1994; 31: 394-400.

45. Beaulieu C, Does MD, Snyder RE, et al. Changes in water diffusion due to Wallerian degeneration in peripheral nerve. Magn Reson Med 1996; 36: 627-31.

46. Basser PJ, Pierpaoli C. Microstructural and physiological features of tissues elucidated by quantitative-diffusion-tensor MRI. J Magn Reson B 1996; 111: 209-19.

47. Minematsu K, Fisher M, Li L, et al. Diffusion-weighted magnetic resonance imaging: rapid and quantitative detection of focal brain ischemia. Neurology 1992; 42: 235-40.

48. Ohgiya Y, Oka M, Hiwatashi A, et al. Diffusion tensor MR imaging of the cervical spinal cord in patients with multiple sclerosis. Eur Radiol 2007; 17: 2499-504.

49. Lin X, Tench CR, Morgan PS, Constantinescu CS. Use of com- 
bined conventional and quantitative MRI to quantify pathology related to cognitive impairment in multiple sclerosis. J Neurol Neurosurg Psychiatry 2008; 79: 437-441.

50. Hiltunen J, Suortti T, Arvela S, Seppa M, Joensuu R, Hari R. Diffusion tensor imaging and tractography of distal peripheral nerves at 3 T. Clinical Neurophysiology 2005; 116: 2315-23.

51. Khalil C, Hancart C, Le Thuc V, Chantelot C, Chechin D, Cotton A. Diffusion tensor imaging and tractography of the median nerve in carpal tunnel syndrome: preliminary results. Eur Radiol 2008; 18: 2283-91.

52. Kabakci N, Gürses B, Firat Z, et al. Diffusion tensor imaging and tractography of median nerve: normative diffusion values. Am J Roentgenol 2007; 189: 923-7

53. MacDonald CL, Dikranian K, Bayly P, Holtzman D, Brody D. Diffusion tensor imaging reliably detects experimental traumatic axonal injury and indicates approximate time of injury. J Neurosci 2007; 27: 11869-76.

54. Fujiyoshi K, Yamada M, Nakamura M, et al. In vivo tracing of neural tracts in the intact and injured spinal cord of marmosets by diffusion tensor tractography. J Neurosci 2007; 27: 11991-8.

55. Yamashita $T$, Kwee TC, Takahara $T$. Whole-body magnetic resonance neurography. N Engl J Med 2009; 361: 538-9.

56. Tsuchiya K, Imai M, Tateishi H, Nitatori T, Fujikawa A, Takemoto S. Neurography of the spinal nerve roots by diffusion tensor scanning applying motion-probing gradients in six directions. Magn Reson Med Sci 2007; 6: 1-5.

57. Takagi T, Nakamura M, Yamada M, et al. Visualization of peripheral nerve degeneration and regeneration: monitoring with diffusion tensor tractography. Neuroimage 2009; 44: 884-92.

58. Jenis LG, An HS. Spine update. Lumbar foraminal stenosis. Spine 2000; 25: 389-94.

59. MacNab I. Negative disc exploration: an analysis of the causes of nerve root involvement in sixty-eight patients. J Bone Joint Surg Am 1971; 53: 891-903.

60. Burton R, Kirkaldy-Willis W, Yong-Hing K, et al. Causes of failure of surgery on the lumbar spine. Clin Orthop 1981; 157: 191-7.

61. Hasegawa $\mathrm{T}, \mathrm{An} \mathrm{H}$, Haughton $\mathrm{V}$, et al. Lumbar foraminal stenosis: critical heights of the intervertebral discs and foramina. J Bone
Joint Surg 1995; 77: 32-8.

62. Kirkaldy-Willis W, Wedge J, Yong-Hing K, et al. Lumbar spinal nerve lateral entrapment. Clin Orthop 1982; 169: 171-8.

63. Vanderlinden RG. Subarticular entrapment of the dorsal root ganglion as a cause of sciatic pain. Spine 1984; 9: 19-22.

64. Herron L. Selective nerve root block in patient selection for lumbar surgery: surgical results. J Spinal Disord 1989; 2: 75-9.

65. Aota Y, Niwa T, Yoshikawa K, et al. Magnetic resonance imaging and magnetic resonance myelography in the presurgical diagnosis of lumbar foraminal stenosis. Spine 2007; 32: 896-903.

66. Krudy AG. MR myelography using heavily $\mathrm{T} 2$-weighted fast spinecho pulse sequences with fat presaturation. Am J Roentgenol 1992; 159: 1315-20.

67. Yamada H, Terada M, Iwasaki H, et al. Improved accuracy of diagnosis of lumbar intra and/or extra-foraminal stenosis by use of three-dimensional MR imaging: comparison with conventional MR imaging. J Orthop Sci 2015; 20(2): 287-94.

68. Iwasaki H, Yoshida M, Yamada H, et al. A new electrophysiological method for the diagnosis of extraforaminal stenosis at L5-S1. Asian Spine J 2014; 8(2): 145-9.

69. Ando M, Tamaki T, Kawakami M, et al. Electrophysiological diagnosis using sensory nerve action potential for the intraforaminal and extraforaminal L5 nerve root entrapment. Eur Spine J 2013; 22: 833-9.

70. Mixter WJ, Barr JS. Rupture of the intervertebral disc with involvement of the spinal canal. N Engl J Med 1934; 211: 210-5.

71. Saritas EU, Cunningham CH, Lee JH, Han ET, Nishimura DG. DWI of the spinal cord with reduced FOV single-shot EPI. Magn Reson Med 2008; 60(2): 468-73.

72. Maki S, Koda M, Ota M, et al. Reduced field-of-view diffusion tensor imaging of the spinal cord shows motor dysfunction of the lower extremities in patients with cervical compression myelopathy. Spine 2015 in press.

Spine Surgery and Related Research is an Open Access article distributed under the Creative Commons Attribution - NonCommercial - NoDerivatives 4.0 International License. To view the details of this license, please visit (https://creativecommons.org/licenses/by - nc - nd/4.0/). 